\title{
Mechanical versus Biological Aortic Valve Implants in the Elderly. A Comparison of Early and Mid-Term Results
}

\author{
LarsI. Thulin, Johan L Sjögren \\ Lund, Sweden
}

\begin{abstract}
Objective - Our aim was to compare, in a non randomized study, the surgical outcome in elderly patients with mechanical (Group 1; $n=83$ ) and bioprosthetic valve implants (Group 2; $n=136$ ).
\end{abstract}

Methods - During a three year period, 219 patients $>75$ years underwent Aortic Valve Replacement. The groups matched according to age, sex, comorbidity, valve pathology and concomitant Coronary Artery Bypass Surgery. Follow-up was a total of 469 patient-years (mean follow-up 2.1 years, maximum 4,4 years).

Results - Operative mortality was zero and the overall early mortality was $2.3 \%$ (within 30 days). Actuarial survival was $87.5 \pm 4.0 \%$ and $66.1 \pm 7.7 \%$ (NS) at 4 years in Group 1 and Group 2, respectively. Freedom from valve-related death was $88.9 \pm 3.8 \%$ in Group 1 and $69.9 \pm 7.9 \%$ (NS) in Group 2 at 4 years.

Conclusion - Aortic Valve Replacement in the elderly (>75 years) is a safe procedure even in cases where concomitant coronary artery revascularization is performed. Only a few anticoagulant-related complications were reported and this may indicate that selected groups of elderly patients with significant life expectancy may benefit from mechanical implants .

Key words: aortic valve, elderly, heart valve prosthesis, cardiac surgery

Heart Valve Section, Division of Heart and Lung Diseases, University Hospital of Lund

Mailing address: Lars I. Thulin - SE-221 85 - Lund, Sweden

E-mail: lars.thulin@thorax.lu.se

Received for publication on 29/1/01

Accepted on 11/4/01
A degenerative aortic stenosis is the most common valve lesion in elderly patients and aortic valve replacement has become the treatment of choice. Because of the demographic changes in Western countries and the advances in surgical techniques and post-operative management, there are an increasing number of elderly patients referred to open heart surgery ${ }^{1}$. At our department there was a 33\% increase in patients over 75 years accepted for aortic valve replacement with or without concomitant revascularization during the study period. Several studies have shown that aortic valve replacement in patients 75 years and older can be performed with an acceptable operative mortality and morbidity ${ }^{2-5}$. In procedures including heart valve replacement, the surgeon must make an important decision between bioprostheses and mechanical heart valves. According to previous studies, age per se, is not a valid criterion for any type of valve. Instead individual factors must be considered ${ }^{6}$. Bioprostheses in elderly are widely accepted and implanted due to freedom of anticoagulant-related adverse events and a low incidence of thrombo-embolism and valve thrombosis ${ }^{4,7}$. Instead there is a higher incidence of re-replacements because of limited durability of the biological valve due to structural deterioration. Patients have an increased life expectancy and there will be a growing number of very old patients facing a re-operation 10-15 years after the initial implantation, with a significant higher operative mortality risk ${ }^{8}$. Recently published studies suggest that mechanical valve prostheses are a safe option in elderly patients. There is a low incidence of thrombo-embolic complications in patients with well-managed anticoagulant therapy. Studies suggest a good quality of life also in patients with a mechanical heart valve prosthesis. Our study was initiated in order to study the early and late results in elderly undergoing aortic valve replacement and to evaluate the results of bioprostheses and mechanical valve implants in patients at age 75 and older.

\section{Methods}

We have performed a retrospective and non randomized study of 219 patients (male 39\%/female $61 \%$ ) who con- 
secutively underwent open heart surgery during a three year period at our institution. The patients were all 75 years and older (mean, 78.6 \pm 2.9 ; range 75-91) at time of surgery and they all had an aortic valve replacement with or without concomitant revascularization. None of the patients had undergone a previous cardiac operation.

The patients were divided in two groups: one group (136 patients) received a biological tissue valve, a pericardial Mitroflow prosthesis (Mitroflow International Inc., Richmond, BC, Canada). The other group, 83 patients, received a mechanical valve implant in the aortic valve position. Three types were used: Sorin Monostrut, 45 patients (Sorin Biomedica Cardio, Saluggia, Italy); CarboMedics bileaflet, 30 patients (Sulzer Carbomedics Inc., Austin, TX, USA) and St. Jude bileaflet (St. Jude Medical Inc., St. Paul, MN, USA) in 8 patients. Whether a patient received a biological valve substitute or a mechanical prosthesis was dependent on the responsible surgeon's personal preferences based on individual patient related factors. The surgical procedures were performed via a median sternotomy and standard techniques in cardiopulmonary by-pass with moderate hypothermia, cold antegrade crystalloid cardioplegia combined with topical cooling using ice slush. All prostheses were implanted with a standard interrupted inversed mattressed suture technique. Operative data are summarized in table I.

The patients' clinical characteristics were collected preoperatively from relevant medical records (Table II). The two groups matched in age, sex, type of aortic valve pathology and severe associated disease. The patients had severe associated diseases in $28 \%$ (23 patients) and 34\% (46 patients) in the mechanical valve and bioprosthetic valve group, respectively. All patients underwent preoperative coronary angiography to confirm any concurrent coronary

\begin{tabular}{|lcc|}
\hline \multicolumn{2}{|c|}{ Table II - Preoperative clinical characteristics of the patients } \\
& $(\mathbf{n = 2 1 9 )}$ & \\
\hline & $\begin{array}{c}\text { Mechanical } \\
\text { implants }\end{array}$ & Bioprostheses \\
& $83(38 \%)$ & \\
\hline $\mathrm{N}^{\text {o }}$ of patients & $32(39 \%)$ & $136(62 \%)$ \\
Sex Male & $51(61 \%)$ & $54(40 \%)$ \\
Female & 78,1 & $82(60 \%)$ \\
Age Mean & 78 & 78,9 \\
& 2,5 & 78 \\
Median SD & $75-86$ & 3,9 \\
Range & $22(27 \%)$ & $48(35 \%)$ \\
80 years or older & $67(81 \%)$ & $115(86 \%)$ \\
Aortic valve lesion & & $2(1 \%)$ \\
Aortic stenosis & $0(0 \%)$ & $19(14 \%)$ \\
aortic insufficiency & $16(19 \%)$ & \\
Combined pathology & & $46(34 \%)$ \\
Preoperative & $23(28 \%)$ & $5(4 \%)$ \\
comorbidity & $2(2 \%)$ & $11(8 \%)$ \\
CVA & $4(5 \%)$ & $10(7 \%)$ \\
DM & $5(6 \%)$ & $4(3 \%)$ \\
Pulmonary insufficiency & $1(1 \%)$ & \\
Carcinoma & $3(4 \%)$ & $(12 \%)$ \\
Arrythmias & $11(13 \%)$ & \\
Miscellaneous & & \\
\hline SD- standard deviation. & & \\
\hline
\end{tabular}

\begin{tabular}{|c|c|c|c|}
\hline \multicolumn{4}{|c|}{ Table I - Operative data } \\
\hline & $\begin{array}{c}\text { Mechanical } \\
\text { implants }(n=83)\end{array}$ & $\begin{array}{l}\text { Bioprostheses } \\
\quad(n=136)\end{array}$ & $p$ value \\
\hline \multicolumn{4}{|l|}{ Valve size (mm) } \\
\hline 19 & 3 & 0 & \\
\hline 21 & 46 & 76 & NS \\
\hline 23 & 26 & 47 & NS \\
\hline 25 & 6 & 13 & NS \\
\hline 27 & 1 & 0 & \\
\hline 29 & 1 & 0 & \\
\hline By-pass time (min) & 111 (SD 37) & 113 (SD 29) & NS \\
\hline $\begin{array}{l}\text { Aortic-clamp } \\
\text { time (min) }\end{array}$ & 81 (SD 22) & 81 (SD 20) & NS \\
\hline Concomitant & & & \\
\hline Revascularization & $31(37 \%)$ & $67(49 \%)$ & NS \\
\hline
\end{tabular}

artery disease. Coronary artery disease (CAD) was defined as a reduction in vessel diameter by at least $50 \%$ on coronary angiography. In the bioprosthetic valve group 49\% (67 patients) of the patients underwent concomitant coronary artery by-pass grafting. Corresponding figure for patients with mechanical valve prostheses was $37 \%$ (31 patients). The concomitant revascularization was performed with a mean of 2,5 grafts and the left internal mammary artery was used in 50 patients $(51 \%)$.

Intravenous continuous heparin anticoagulation therapy was started on the first postoperative day in all patients. Heparin was administrated until the prothrombin time could be adjusted by daily warfarin administration. The recommended anticoagulation level was a prothrombin time of $15-25 \%$ (international normalized ratio, INR 2.83 to 1.95). Patients with a bioprosthesis were treated with warfarin for 3 months postoperatively (unless atrial fibrillation or other reasons indicated prolonged anticoagulant therapy). After discharge, the referring physician or an anticoagulationreception at the hospital, staffed with authorized nurses, made the routine check-up and administration of warfarin according to the anticoagulation protocol.

All the patients' records were checked to obtain information on any possible complication. The follow-up was $100 \%$ complete and represented 469 patient-years with a mean of $2.4 \pm 1.3$ years in the mechanical valve group and $2.0 \pm 1.2$ years in patients with a biological valve implant. Maximum follow-up time was 4.4 years. We used the guidelines of The Society of Thoracic Surgeons and The American Association for Thoracic Surgery for the definitions of morbid events and mortality ${ }^{9}$.

Statistical analysis used were chi-square test and Student's t-test for inter-group comparisons. Actuarial survival estimates were calculated and plotted according to the Kaplan-Meier method. The log-rank test (Cox-Mantel) was used for comparison of the actuarial overall survival and event-free curves, respectively, between the two groups.

\section{Results}

The overall early mortality (within 30 days) was $2.3 \%$. 
Early mortality was $4.8 \%$ (4/83) in patients with mechanical valves and $0.7 \%$ (1/136) in patients with a bioprosthetic valve implant. The causes of in-hospital death in the mechanical implant group were: two patients with post-operative myocardial infarction, one patient with myocardial infarction and progressive heart failure, and one patient with multiple organic failure. There were only one early death in the group of patients who received a bioprosthesis. This patient died from aortic dissection at the cannulation site resulting in heart tamponade. The diagnosis was confirmed at autopsy. There was no significant difference in length of hospital stay between the two groups. Patients stayed at the intensive care unit for mean 3.45 days. The median length of total post-operative stay at the hospital before discharge was $13.3 \pm 5.1$ days in the mechanical valve group and $13.7 \pm 4.6$ days in the biological valve group.

After hospital discharge and within the follow-up time, 5 patients died in the mechanical valve group and 20 patients died in the bioprosthetic valve group. There were 4 cardiac-related deaths in the mechanical valve implant group: heart failure in 2, myocardial infarction in 1 (not classified as a valve-related death due to the patients concomitant coronary disease) and anticoagulant-related hemorrhage (cerebrovascular accident) in 1 patient. Non cardiac-related death occurred in 1 patient with urosepsis and subsequently multiple organic failure. In the biological valve group 11 deaths were due to cardiac causes: heart failure in 4, myocardial infarction in 2 (none of these two deaths were considered valve-related due to the angiographic findings of coronary lesions or the presence of angina pectoris) and cerebrovascular accidents in 4 patients. One (1) patient with a Mitroflow bioprosthesis developed an endocarditis and was readmitted after 4 months. This patient underwent reoperation and had the biological valve replaced for a mechanical implant, but the patient later succumbed from postoperative cardiogenic shock. Other non cardiac causes of late deaths were: carcinoma in 4, sepsis in 2, strangulation ileus in 1, gastrointestinal bleeding (no anticoagulation) in 1 and a ruptured abdominal aneurysm in 1 patient. The actuarial survival, including early mortality, at 53 months was $87.5 \pm$ $4.0 \%$ and $66.1 \pm 7.7 \%$ respectively in the mechanical and the bioprosthetic valve group. There was no significant difference between the two groups, although a tendency towards better survival in the mechanical group. Freedom from valverelated death was $88.9 \pm 3.8 \%$ and $69.9 \pm 7.9 \%$ respectively in the mechanical and the bioprosthetic valve group (Fig.1) This was not a statistically significant difference.

No patient from the mechanical valve group had a late non-fatal complication such as thromboembolic event, anticoagulant-related hemorrhage or prosthetic valve endocarditis. Furthermore, no late reoperation was performed in this group due to valvular dysfunction or for any other reason.

A cerebrovascular accident without permanent deficit occurred 24 months after implantation in one (1) patient with a bioprosthesis (no present anticoagulant therapy).

No anticoagulant-related hemorrhage was reported in patients with biological implants.

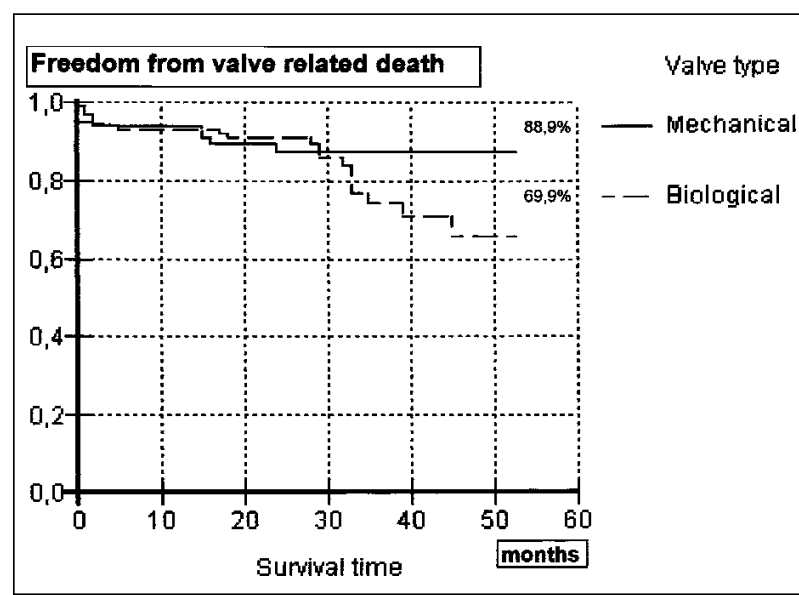

Fig 1 - Freedom from valve-related death in patients with mechanical and bioprosthetic valves.

One patient with a bioprosthesis had a re-replacement after four months due to endocarditis (see above 3.2.). This patient later succumbed from cardiogenic shock.

\section{Discussion}

The choice between different types of valve implants is an important issue and there has been several studies trying to decide whether one should use a mechanical or a biological valve substitute in elderly patients ${ }^{10,11}$. Still there is no consensus on this matter. This is not unique only in elderly patients ${ }^{12}$. With advanced age the choice is becoming more crucial, since negative consequences and related complications are less well tolerated by the aged organism. There are diminished marginal in several organ systems in the elderly patient, due to the normal changes associated with senescence ${ }^{13}$. These patients with most of their lives behind them, have less margins when undergoing surgical procedures and if there should be an intervention with good long-term outcome and a good quality of life without impaired autonomy, one should make a selection based on individual characteristics and not on old age alone ${ }^{14}$. Most elderly people prefer a good quality of life to longer lifespan. Even the very old patient with AVR has shown to benefit from surgery with an improvement in heart symptoms and quality of life ${ }^{15}$. What type of valve implant will the individual elderly patient finally benefit from most?

With increasing life expectancy in the elderly, the use of mechanical valve prostheses may be the best choice for a selected group of patients ${ }^{16-18}$. Mechanical valves will require a mandatory life-long anticoagulant therapy. Several studies claim that when properly managed, elderly patients receiving warfarin appear to have no greater risk for hemorrhagic complications than do younger patients ${ }^{19,20}$. A current issue of discussion is whether one could optimize the anticoagulant therapy in elderly using lower doses of warfarin ${ }^{21,22}$. Even self-management of anticoagulation following patient education has shown to be applicable in selected elderly patients. Furthermore, recent studies have sho- 
wn that the benefit of implanting bioprosthesis not requiring anticoagulation, does not outweigh the risk of its limited durability due to structural deterioration, with a prohibitive risk of re-replacement ${ }^{6}$.

This study reports an overall early mortality of $2.3 \%$, which clearly shows that aortic valve replacement can be safely performed in elderly patients. These figures are similar or even lower compared to previously published studies, especially considering the fact that $44.7 \%$ in the study population had a combined surgical procedure with concomitant revascularization.

The fact that all patients underwent preoperatively coronary angiography, regardless of presence of overt signs of $\mathrm{CAD}$, has likely resulted in the high percentage of concomitant CABG. It may be argued that the relatively low incidence both peri- and postoperative deaths are a result of our aggressive attitude towards CAD. It is interesting to notice that in this non randomized study (but with no preoperative differences in patient related data) a higher early mortality in the mechanical group was "compensated" by an increased mortality in the bioprosthetic group at follow up after up till 4.4 years.

In our population, there was no significant difference between the two groups in terms of associated diseases or concomitant revascularization. There was a tendency in the patients who received a bioprosthesis of having a higher percentage of concomitant coronary artery disease. There was no significant difference in length of stay at the intensive care unit nor at the general ward.

The actuarial overall survival figures were not significantly worse in the mechanical valve group despite the imposed and potentionally dangerous anticoagulation thera- py. A possible explanation for this finding is that the surgeon selects patients according to estimated life expectancy and makes a decision in favor of mechanical valve implants. This group of patients will probably live longer and be in such a condition that chronic anticoagulation will be no major obstacle. This may also be reflected in the event-free survival favoring the choice of mechanical prostheses.

The fact that there was a low incidence of reported complications from anticoagulant treatment, both fatal and nonfatal, may be a result of accurate management of the warfarin protocol. Patients receive substantial information on chronic anticoagulation and a well functioning system of outpatient care and control of PT or INR levels is mandatory. All complications were documented and even minor episodes of bleeding or signs of systemic embolisation were reported.

There is always a potential risk of bias and underestimating the frequency of non-fatal anticoagulant-related events, even though the control system and documentation were of high quality. It is important and necessary to adopt the reported results with care and cautiously consider its implications.

In conclusion, this study shows that AVR may be performed safely in elderly patients and even in patients on a life-long anticoagulant therapy, the incidence of valve-related complications may be low. The results in this report suggest that elderly patients can benefit from mechanical valves taking individual patient characteristics into careful consideration accomplishing a thorough risk assessment. Furthermore, our findings imply strongly that there should be no general recommendation of valve type to be implanted based on age alone.

\section{References}

1. Gehlot A, Mullany CJ, Ilstrup D, et al. Aortic valve replacement in patients aged 80 years and older: early and long-term results. J Thorac Cardiovasc Surg 1996; 111: 1026-36.

2. Thulin L, Sjögren J. Open-heart surgery in a growing geriatric population: patient selection and risk factors to be considered. Cor Artery Dis 1998; 9: 365-372.

3. Katz NM, Hannan RL, Hopkins RA, Wallace RB. Cardiac operations in patients aged 70 years and over: mortality, length of stay, and hospital charge. Ann Thorac Surg 1995; 60: 96-101.

4. Holper K, Wottke M, Lewe T. Bioprosthetic and mechanical valves in the elderly: benefits and risks. Ann Thorac Surg 1995; 60: S443-6.

5. Culliford A, Galloway A, Colvin S, Grossi E, Baumann F, Esposito R et al. Aortic valve replacement for aortic stenosis in persons aged 80 and over. Am J Cardiol 1991; 67: 1256-60.

6. Arom KV, Emery RW, Nicoloff DM, Petersen RJ. Anticoagulant related complications in elderly patients with St. Jude mechanical valve prostheses. J Heart Valve Dis 1996; 5: 505-10.

7. Myken PSU, Caidahl K, Larsson P, Larsson S, Wallentin I, Berggren HE. Mechanical versus biological valve prosthesis: a 10-year comparison regarding function and quality of life. Ann Thorac Surg 1995; 60: S447-52.

8. Tyers GFO, Jamieson WRE, Munro AI. Reoperation in biological and mechanical valve populations: fate of the reoperative patient. Ann Thorac Surg 1995; 60: S464-9.

9. Edmunds LH, Clark RE, Cohn LH, Miller DC, Wiesel RD. Guidelines for reporting morbidity and mortality after cardiac valvular operations. Ann Thorac Surg 1988; 46: 257-9.

10. Loisance DY, Mazzocotelli JP, Bertrand PC, Deleuze PH, Cachera JP. Mitroflow pericardial valve: long-term durability. Ann Thorac Surg 1995; 56: 131-6.

11. MoggioRA, Pooley RW, Sarabu MR, Christiana J,Ho AW, Reed GE. Experience with the Mitroflow aortic bioprosthesis. J Thorac Cardiovasc Surg 1994; 108: 215-20.
12. Mohl W, Kilo J, Moidl R, Rödler S, Wolner E. Standards in valve replacement. Which Artificial valve is really a standard? Cor Europaeum 1997; 6: 10-13.

13. Rowe JW. Health care of the elderly. NEngl J Med 1985; 312: 827-35.

14. Kirsch M, Guesnier L, LeBesnerais P, Hillion M-L, Debauchez M, Seguin J, Loisance DY. Cardiac operations in octogenarians: perioperative risk factors for death and impaired autonomy. Ann Thorac Surg 1998; 66: 60-7.

15. Levin I, Olivecrona G, Thulin L, Olsson B. Aortic valve replacement in patients older than 85 years: outcomes and their effects on their quality of life. Coronary Artery Disease 1998, 9: 373-80.

16. DebetazLF, Ruchat P, Hurni M, etal. St. Jude Medical prosthesis: an analysis of longterm outcome and prognostic factors. J Thorac Cardiovasc Surg 1997; 113: 134-48.

17. Horstskotte D, Schulte HD, Bircks W, Stauer B. Lower intensity anticoagulation therapy results in lower complication rates with the St. Jude Medical prosthesis. J Thorac Cardiovasc Surg 1994; 107: 1136-4.

18. Ninet J, Tronc F, Robin J, Aleksic AC, Champsaur G. Mechanical versus biological isolated aortic valvular replacement after the age of 70: equivalent longterm results. Eur J Cardiothorac Surg 1998; 13: 84-9.

19. Fihn SD, Callahan CM, Martin DC, McDonell MB, Henikoff JG, White RH. The risk for and severity of bleeding complications in elderly patients treated with warfarin. Ann Intern Med 1996; 124: 970-79.

20. HirshJ,DalenJE, DeykinD, PollerL. Oral anticoagulants: mechanismof action, clinical effectiveness, and optimal therapeutic range. Chest 1992; 102(Suppl): 312-26S.

21. Cannegieter SC, Rosendaal FR, Wintzen AR, van der Meer FJ, Vandenbroucke JP, Briët E. Optimal oral anticoagulant therapy in patients with mechanical heart valves. N Engl J Med 1995; 333: 11-7.

22. Stein PD, Alpert JS, Copeland J, Dalen JE, Goldman S, Turpie AGG. Antithrombotic therapy in patients with mechanical and biological prosthetic heart valves. Chest 1992; 102(Suppl): 445S-55S. 